\title{
Application of GEE Models for Assessing Maternal Health Complications
}

\author{
Mohammad Shaha Alam Patwary ${ }^{1}$, Soma Chowdhury Biswas ${ }^{2}$ \\ ${ }^{1}$ Department of Mathematics, Statistics, and Actuarial Science, Butler University, Indianapolis, IN, USA \\ ${ }^{2}$ Department of Statistics, University of Chittagong, Chittagong, Bangladesh \\ Email: mpatwary@butler.edu,soma_chow@hotmail.com
}

How to cite this paper: Patwary, M.S.A. and Biswas, S.C. (2021) Application of GEE Models for Assessing Maternal Health Complications. Applied Mathematics, 12, 563-575. https://doi.org/10.4236/am.2021.127040

Received: May 31, 2021

Accepted: July 4, 2021

Published: July 7, 2021

Copyright $\odot 2021$ by author(s) and Scientific Research Publishing Inc. This work is licensed under the Creative Commons Attribution International License (CC BY 4.0).

http://creativecommons.org/licenses/by/4.0/ (c) (i) Open Access

\begin{abstract}
Bangladesh, a developing country, gained success towards the fifth-millennium development goals target of reducing its maternal mortality ratio by three quarters by 2015, but yet worked more on it for further reduction of maternal mortality. In this light, though Bangladesh is committed to the sustainable development goals target of reducing its maternal mortality ratio to be reduced from 170 to 105 per 100,000 live births, the scope of research on this issue is limited because the maternal morbidity data is scarce in Bangladesh. In this paper, the prospective data on maternal morbidity in rural Bangladesh (collected by BIRPERHT) have been employed to trace out the high-risk and life-threatening factors associated with pregnancy-related complications. The subject-specific generalized estimating equations (SS-GEE) model with random effect structure is used for multivariate binary data for the repeated observations. The findings indicate that the risk of suffering from pregnancy complications is higher for high economic status, lower age at marriage, not visited for medical check-ups, outside home workers, and having miscarriage or abortion. Comparing the SS-GEE model with other correlation structures and relative efficiency factors, the SS-GEE model with random effect structure is well fitted for the prospective repeated observation data.
\end{abstract}

\section{Keywords}

Multivariate Binary Response, Repeated Observations, GEE, Random Effect, Pregnancy Complications

\section{Introduction}

Bangladesh, a south Asian developing country with a lot of economic and health-related challenges and contingencies, was committed to target of reduc- 
ing its maternal mortality rate by 75 percent between 1990 and 2015 to the fifth Millennium Development Goals (MDG-5) [1]. After gaining remarkable success in the reduction of maternal mortality in the MDG-5, Bangladesh is now focused on a set of targets of the sustainable development goals (SDGs). Multiple of these targets are closely related to newborn, child, and maternal health [2] [3]. In this light, Bangladesh is committed to the target of reducing its maternal mortality ratio from 170 to 105 per 100,000 live births [4] with the predicted mortality ratio of 87.3 in 2021 [2]. However, antenatal care, professional attendance at delivery and postnatal care services are most significant determinants for reducing such a rate and preventing infant mortality as well. Moreover, these services are inadequately developed in the least developed countries like, Bangladesh. Utilization of antenatal care services amplified significantly, from 24 percent in 1991 to 60 percent in 2004 [5]. In [6] it is reported that female literacy is expected to be a very prolific strategy to change the tide of maternal mortality. Antenatal care is an important predictor of safe delivery and provides health information and services that can improve maternal and infants health [7] [8]. Economic barriers to utilization of facility-based care are outlawed among the poor, even where the actual care is free-of-cost [9] [10] [11]. However, in [12], it has been observed that cultural issues, distance, infrastructure and socio-economic status are important determinants of maternal healthcare-seeking behavior. It is extracted that preceding maternal deaths, most of the pregnancies are attended either by traditional practitioners or are not attended at all [13]. The levels of maternal mortality in Bangladesh are remarkably low given the extremely low levels of uptake of maternity care [14]. Regardless of improvements, pregnancy-related complications remain the leading cause of maternal death and disability among women of childbearing age, irrespective of different indigenous, non-indigenous and cultural groups [15]. In Bangladesh, the most important determinants of maternal mortality are eclampsia, septic abortion, postpartum sepsis, obstructed labor and antepartum and postpartum hemorrhage [16] [17] [18]. Severe anemia boosts up the risk of mortality while there is a poor evidence of increased risk associated with moderate anemia [19]. In India, 18 percent of the women reported complications during antenatal period and an equal proportion during childbirth [20]. The most common likelihood-based methods for analyzing multivariate binary data are multivariate probit and multivariate logit models that consider univariate normal and logistic distributions as univariate margins respectively [21]. On the other hand, the generalized estimating equations (GEE) methodology, an estimating equation based method, proposed in [22] is extensively used for analyzing multivariate binary response data [23]. In [24], it has been observed that the estimator of the correlation based on conditional residuals yielded more efficient estimates than the usual GEE estimator that was based on unconditional residuals. In another study, the estimates obtained were very analogous to the results obtained by using a weighted least squares (WLS) methodology [25]. The GEE estimates for Food supplement, economic status, wanted pregnancy, gainful employment, education, number of pregnancies, and 
age at marriage obtained under the assumption of exchangeable correlation provide more efficient estimators as compared to the independence and autoregressive correlation structures [26]. Utilizing GEE method, authors in [27] observed that taking special food during the pregnancy period reduces the number of complication irrespective of different correlation structures. Age at marriage has a significant effect on pregnancy related complications [28]. The risk of suffering from complications is extensively higher among the women in case of unwanted pregnancy, lower level or no schooling, lower age at marriage ( $<15$ years), 5 or more pregnancies prior to the index pregnancy [29]. In this paper, an attempt is made to spot out the covariates entangled with some life-threatening and high-risk complications that sandwich among the rural women during antenatal period with an application to random effect Subject Specific-GEE (SS-GEE) model.

This paper is outlined in four different sections where materials and methods are discussed in Section 2. Section 3 focuses on results and discussion, and the paper is concluded with the conclusions section (Section 4).

\section{Materials and Methods}

\subsection{Data}

The primary source of data for this study was taken from Bangladesh Institute of Research for Promotion of Essential and Reproductive Health and Technologies (BIRPERHT) survey on maternal morbidity in the rural areas of Bangladesh. With the motive to identify the risk factors for maternal morbidity in Bangladesh, BIRPERHT conducted this survey and collect prospective repeated observation data. All the pregnant women of duration at most six months of the selected unions comprised the sample. All the selected women were followed till 90 days after delivery. The data on socio-economic, background, pregnancy-related care and practice, extent of morbidity during the index pregnancy, delivery and postpartum period or abortion were collected on 1020 women. Out of these 1020 women, 993 had at least one antenatal follow-up, and 1005 had information on pregnancy termination. Finally, 1006 had at least one postpartum follow-up. Since progressively large proportions of selected pregnant women were lost to follow after 4 follow-ups, we consider up to 4 follow-ups for the present dissertation. Table 2 shows the frequency and percentage distribution of the respondents interviewed during each follow-up.

\subsection{Variables}

The factors that contribute to maternal morbidity are too numerous to enumerate. The causes are multiple, interrelated and tiered. Information on socio-economic and demographic characteristics, pregnancy related care and practice, morbidity during the period of follow-up as well as in the past, information concerning complications at the time of delivery and during the postpartum period, etc. were also collected for all the selected pregnant women. 
In the light of the objectives of this study, pregnancy complication variable is computed by gathering the major life-threatening antenatal complications hemorrhage, oedema, excessive vomiting, and fits or convulsion. In this study the response variable is considered as binary taking the value 1 if at least one of the complications was present. Notationally, it can be defined as:

$$
y= \begin{cases}1, & \text { if the women suffers at least one of the major complications } \\ 0, & \text { otherwise }\end{cases}
$$

Among the available covariates, only five important covariates are considered in this study, which are: economic status of the respondents, age at marriage, antenatal check-up, place of work, and any miscarriage or abortion on the basis of significance probability ( $\mathrm{p}$-value $<0.05$ ) which are supported by the studies from [24] [25] [26] [27]. All these covariates are coded as binary with the reference categories, less than average for economic status, less than 16 years for age at marriage, not visited for antenatal check-up, at home for place of work, and no for miscarriage or abortion, respectively.

\subsection{Methods}

In this study, subject specific random effect model [30] [31] [32] is applied to observe the association between certain covariates with repeated observations on status of complications at different follow-ups. Along with many other advantages, subject specific random effect model offers consistent estimates of parameters irrespective of the underlying true correlation structure, but may be inefficient when the true correlation structure is not correctly specified [33]. The parameter estimates are also sensitive to outliers [33] [34]. Here, generalized estimating equation [22] [23] has been adapted to the data of one to four repeated binary observations of the mother registered at BIRPERHT and we have used statistical programming language $\mathrm{R}$ version 4.0 .5 to analyze the data and to get the estimates.

Suppose we are in a setting with $m$ subjects (or clusters) and $n_{i}$ observations per subject, where $\boldsymbol{Y}_{i}=\left(Y_{i 1}, Y_{i 2}, \cdots, Y_{i n_{i}}\right)^{\prime}, i=1, \cdots, m$ is a $n_{i} \times 1$ vector of dichotomous response, $\boldsymbol{X}_{i}=\left(X_{i 1}, X_{i 2}, \cdots, X_{i p}\right)$ be an $n_{i} \times p$ design matrix for $p$ covariates, then the general mixed model [30] [31] [32] [35] for ith subject is given by $\boldsymbol{Y}_{i}=\boldsymbol{X}_{i} \boldsymbol{b}+\boldsymbol{Z}_{i} \boldsymbol{v}_{i}+\boldsymbol{\epsilon}_{i}$ which satisfies $\boldsymbol{Y}_{i} \mid \boldsymbol{v}_{i} \sim \mathcal{N}\left(\boldsymbol{X}_{i} \boldsymbol{b}+\boldsymbol{Z}_{i} \boldsymbol{v}_{i}, \sigma^{2} \boldsymbol{I}_{i}\right)$, $\boldsymbol{v}_{i} \sim \mathcal{N}(\mathbf{0}, \boldsymbol{D}), \boldsymbol{\epsilon}_{i} \sim \mathcal{N}\left(\mathbf{0}, \sigma^{2} \boldsymbol{I}\right)$. The $\boldsymbol{b}$ and $\boldsymbol{v}_{i}$ are coefficient of fixed effects and random effects, respectively. The random effects coefficients, $\boldsymbol{v}_{i}$, are used to model the random variation in the $\boldsymbol{Z}_{i}$ covariates at different levels of the data. It also represents the deviation of the intercept of a specific subject from the average intercept in the group to which that subject belongs. The random intercept model implies marginal compound-symmetry model where the effect of the random intercept is absorbed in the marginal covariance structure.

It is hypothesized that the underlying distribution for random effects in the model that serves as the genesis of the within-panel correlation. In other words, it can be said that the correlation among individual responses within the same 
subject (patient) is accounted for by adding a subject-specific random effect term to the logit.

There are three items we must address to build models for SS-GEE: 1) a distribution for the random effect, 2) the expected value which depends on the link function and the distribution of the random effect, 3) the variance function of the usual variance and the random effect. This formulation assumes a single random effects coefficient, $v_{i}$, follows the Gaussian distribution with mean zero and variance, $\sigma_{v}^{2}$. The expression for the marginal mean for the logit link has no closed form solution, but can be approximated by

$$
\begin{aligned}
\mu_{i t} & =\int g^{-1}\left(x_{i t} \beta+v_{i}\right) \mathrm{d} F v_{i} \\
& =\int \frac{\exp \left(x \beta+v_{i}\right)}{1+\exp \left(x \beta+v_{i}\right)} \frac{1}{\sqrt{2 \pi \sigma_{v}^{2}}} \exp \left(-\frac{v_{i}^{2}}{2 \sigma_{v}^{2}}\right) \mathrm{d} v_{i} \\
& \approx \phi\left(\frac{x_{i t} \beta}{\sqrt{1+c^{2} \sigma_{v}^{2}}}\right), \text { where } c=\frac{16 \sqrt{3}}{15 \pi} ;
\end{aligned}
$$

and variance-covariance by

$$
\begin{aligned}
\mathbb{V}\left(y_{i t}\right)= & \int\left[g^{-1}\left(x_{i s} \beta+v_{i}\right)-\mu_{i s}\right]\left[g^{-1}\left(x_{i t} \beta+v_{i}\right)-\mu_{i t}\right] \mathrm{d} f\left(v_{i}\right) \\
& +a(\Phi) I(s=t) \int \mathbb{V}\left(\mu_{i t}\right) \mathrm{d} f\left(v_{i}\right)
\end{aligned}
$$

According to [36], the approximation of the variance-covariance matrix is obtained by

$$
\begin{aligned}
& \boldsymbol{V}_{i}=\mathbb{V}\left(y_{i}\right) \approx \mathbb{V}\left[g^{-1}\left(x_{i t} \beta\right)+\frac{\partial}{\partial v_{i}} g^{-1}\left(x_{i t} \beta+v_{i}\right)\right] \\
&+\Phi \mathbb{E}\left\{\mathbb{V}\left[g^{-1}\left(x_{i t} \beta\right)+\frac{\partial}{\partial v_{i}} g^{-1}\left(x_{i t} \beta+v_{i}\right)\right]\right\} \\
& \approx D\left(\frac{\partial \mu}{\partial \eta}\right)^{2} \sigma_{v}^{2}+\Phi \mathbb{V}(\mu) J J^{\mathrm{T}}=\tilde{\mathbb{V}}\left(y_{i}\right)=\tilde{V}_{i}
\end{aligned}
$$

where $J$ is a vector of indicator variables.

The first term is a matrix of variance components for the random effect and the second term is a matrix of the dispersion parameter, and where, mean vector $\mu_{i t}=g^{-1}\left(x_{i t} \beta+v_{i}\right)$ and the logit, $\eta_{i t}=\log \left(\frac{\mu_{i t}}{1-\mu_{i t}}\right)=x_{i t} \beta+v_{i}$. Using $\log \left(\frac{\mu_{i t}}{1-\mu_{i t}}\right)=\eta_{i t}$, we have, $\mu_{i t}=\frac{\exp \left(\eta_{i t}\right)}{1+\exp \left(\eta_{i t}\right)}$ and $\frac{\partial \mu_{i t}}{\partial \eta}=\frac{\partial\left(\frac{\exp \left(\eta_{i t}\right)}{1+\exp \left(\eta_{i t}\right)}\right)}{\partial \eta_{i t}}=\frac{\exp \left(\eta_{i t}\right)}{\left(1+\exp \left(\eta_{i t}\right)\right)^{2}}$, where $\eta=x_{i t} \beta+v_{i}, i=1, \cdots, m ; t=1, \cdots, n_{i}$.

According to [36], the expression for $\mathbb{V}\left(y_{i t}\right)=a(\Phi) \mathbb{V}\left(\mu_{i t}\right)$ is nothing but the diagonal matrix $\mathbb{V}\left(y_{i t}\right)=\operatorname{diag}\left[\pi\left(x_{i j}\right)\left(1-\pi\left(x_{i j}\right)\right)\right]$, where $\mu_{i t}=\pi\left(x_{i j}\right)$ due to [22]. 
To estimate the parameters of SS logistic regression model by the method of GEE we put $\mu_{i t}$ and $\boldsymbol{V}_{i}=\tilde{\boldsymbol{V}}_{i}$ to the generalized estimating equation of [22] given by $\sum_{i=1}^{m} \boldsymbol{D}_{i}^{\prime} \boldsymbol{V}_{i}^{-1} \boldsymbol{S}_{i}=\mathbf{0}$, where $\boldsymbol{D}_{i}=\boldsymbol{A}_{i}^{\prime} \boldsymbol{X}_{i}, \quad \boldsymbol{X}_{i}$ is the $n_{i} \times(p+1)$ matrix of covariates and $\boldsymbol{S}_{i}$ is the vector with $t$ th element of the residual $s_{i j}=y_{i j}-\pi\left(x_{i j}\right)$. Then, solve for $\boldsymbol{\beta}$ to get the estimates of SS-parameters $\left(\boldsymbol{\beta}^{\text {SS }}\right)$.

In practice, the random effect terms are unobserved and this leads to complications when we consider estimation of the regression coefficients $\boldsymbol{\beta}^{\text {SS }}$ and the estimate of the variance-covariance matrix of the estimated SS-parameters is given by

$$
\begin{aligned}
\hat{\boldsymbol{V}}_{\boldsymbol{\beta}=\hat{\boldsymbol{\beta}}}= & \left(\sum_{i=1}^{m} \frac{\partial \hat{\boldsymbol{\mu}}_{i}^{\prime}}{\partial \boldsymbol{\beta}} \tilde{\boldsymbol{V}}_{i}^{-1} \frac{\partial \hat{\boldsymbol{\mu}}_{i}}{\partial \boldsymbol{\beta}}\right)^{-1}\left(\sum_{i=1}^{m} \frac{\partial \hat{\boldsymbol{\mu}}_{i}^{\prime}}{\partial \boldsymbol{\beta}} \tilde{\boldsymbol{V}}_{i}^{-1}\left(\boldsymbol{Y}_{i}-\hat{\boldsymbol{\mu}}_{i}\right)\left(\boldsymbol{Y}_{i}-\hat{\boldsymbol{\mu}}_{i}\right)^{\prime} \tilde{\boldsymbol{V}}_{i}^{-1} \frac{\partial \hat{\boldsymbol{\mu}}_{i}}{\partial \boldsymbol{\beta}}\right) \\
& \times\left(\sum_{i=1}^{m} \frac{\partial \hat{\boldsymbol{\mu}}_{i}^{\prime}}{\partial \boldsymbol{\beta}} \tilde{\boldsymbol{V}}_{i}^{-1} \frac{\partial \hat{\boldsymbol{\mu}}_{i}}{\partial \boldsymbol{\beta}}\right)^{-1}
\end{aligned}
$$

In the final stage, superiority of the model has been assessed by means of kappa-like statistic [37] [38] [39] and very well known relative efficiency of estimators.

\section{Results and Discussion}

Respondents entered into the study at different times throughout their pregnancies for follow-ups. The study found that the number of respondents decreased sharply after the first two follow-ups. The most remarkable feature of different surveys conducted in South Asian countries is that the towering majority of respondents undergo some identifiable problem or illness during pregnancy or during labor and delivery.

Table 1 depicts the distribution of respondents by complications during pregnancy and by the selected characteristics. It is observed that about 25 percent

Table 1. Percentage distribution of selected socio-demographic covariates by complications during pregnancy of the selected respondents.

\begin{tabular}{cccccc}
\hline \multirow{2}{*}{ Covariate } & Category & \multicolumn{2}{c}{ Pregnancy complication } & \multirow{2}{*}{$\chi^{2}$ Statistic (df) } & p-value \\
\cline { 3 - 4 } & & No & Yes & & \\
\hline \multirow{2}{*}{ Economic status } & Low & 75.3 & 24.7 & $6.99(1)$ & 0.008 \\
& High & 70.5 & 29.5 & & \\
Age at marriage & $\leq 15$ years & 70.2 & 29.8 & $6.81(1)$ & 0.009 \\
& $>15$ years & 74.6 & 25.4 & & \\
Antenatal check-up & Not visited & 53.1 & 46.9 & $245.05(1)$ & $<0.001$ \\
& Visited & 79.8 & 20.2 & & \\
Place of work & At home & 72.1 & 27.9 & $12.17(1)$ & $<0.001$ \\
& Outside & 54.8 & 45.2 & & \\
Any miscarriage or abortion & No & 72.4 & 27.6 & $4.62(1)$ & 0.032 \\
& Yes & 67.7 & 32.3 & & \\
\hline
\end{tabular}


of the respondents of low economic status suffer from one of the pregnancy complications as compared to 29.5 percent of the respondents of high economic status (Table 1). Although it is surprising that respondents belonging to higher socio-economic status have reported higher percentage with complications during pregnancy, there might be some explanations. Women from lower socio-economic status are exposed to more physical labor during pregnancy that might help them to avoid some of the complications. It is also observed that the prevalence of complications is relatively low for those with age at marriage 16 years or higher as compared to those who reported their age at marriage less or equal to 15 years. In rural areas of Bangladesh, the gap between the age at marriage and birth of first child is not much.

It is ideal to state that those who got married at very young age begin their childbearing at a younger age are physiologically unfit to become a mother. The respondents who did not attend the antenatal check-up suffered from complications at a higher proportion ( 47 percent) than those who attended the check-ups (20.2 percent). Table 1 also presents that the proportion of respondents with complications decreases with level of their working place. Among the respondents who work at home, 27.9 percent reported complications during pregnancy, whereas 45.2 percent of the respondents working outside of home encountered pregnancy complications. This may be considered as the reflector of the fact that women having safe and comfortable working place can avoid some complications during pregnancy as compared to their counterparts. The high-risk group women are those who have had experienced any miscarriage or abortion. These types of deliberate or accidental hazards always accelerate the risk of complications for the reproductive women. It is observed that the life-threatening or high-risk complications are prevalent at a higher proportion among the high-risk groups. Thirty two percent of the women with any miscarriage or abortion reported to have complications as compared to that of 27.6 percent among those who do not experience any miscarriage or abortion. The p-values presented in Table 1 are significantly smaller than 0.05 which indicates the significant association between the covariates and pregnancy complication.

Table 2 represents the follow-up distribution of respondents with or without complications. We observe that the number of respondents decreases rapidly as

Table 2. Distribution of respondents interviewed during selected follow-up period by complications status.

\begin{tabular}{ccccccc}
\hline & \multicolumn{6}{c}{ Complications } \\
\cline { 2 - 7 } Follow-up & \multicolumn{2}{c}{ No } & \multicolumn{2}{c}{ Yes } & \multicolumn{2}{c}{ Total } \\
\cline { 2 - 7 } & Frequency & Percentage & Frequency & Percentage & Frequency & Percentage \\
\hline 1 & 592 & 59.7 & 400 & 40.3 & 992 & 97.3 \\
2 & 678 & 73.9 & 239 & 26.1 & 917 & 89.9 \\
3 & 599 & 77.7 & 172 & 22.3 & 771 & 75.6 \\
4 & 478 & 80.5 & 116 & 19.5 & 594 & 58.2 \\
\hline
\end{tabular}


number of follow up increases. However, the distribution of available respondents shows that at the first follow up more than 40 percent had one or more complications.

In this part of the analysis, we have presented the estimates of the coefficients of the covariates obtained under the assumption of different structural relationship within the responses. The following tables (Tables 3-5) provide the estimates of the parameters of the link function along with their estimated standard error, associated Wald test, odds ratio, Chi-square test, and kappa-like statistic.

Table 3. GEE estimates for maternal morbidity under the assumption of working independence within the responses.

\begin{tabular}{cccccc}
\hline Covariate & Coefficient & Standard error & Wald Statistic & p-value & Odds ratio \\
\hline Constant & -0.0387 & 0.0312 & -1.2404 & 0.218 & 0.9620 \\
Economic status & 0.5513 & 0.2306 & 2.3907 & 0.017 & 1.7355 \\
Age at marriage & -0.1079 & 0.0250 & -4.3160 & $<0.001$ & 0.8977 \\
Antenatal check-up & -0.2908 & 0.0546 & -5.3260 & $<0.001$ & 0.7477 \\
Place of work & 0.2991 & 0.1056 & 2.8324 & 0.005 & 1.3486 \\
Any miscarriage or abortion & 0.2018 & 0.053 & 3.7932 & $<0.001$ & 1.2236 \\
\hline
\end{tabular}

Chi-Square Value $=532.1083(\mathrm{p}$-value $<0.001) ;$ Kappa-like statistic $=0.527$.

Table 4. GEE estimates for maternal morbidity under the assumption of exchangeable correlation within the responses.

\begin{tabular}{cccccc}
\hline Covariate & Coefficient & Standard error & Wald Statistic & p-value & Odds ratio \\
\hline Constant & -0.0413 & 0.0149 & -2.7718 & 0.006 & 0.9595 \\
Economic status & 0.5340 & 0.0828 & 6.4493 & $<0.001$ & 1.7057 \\
Age at marriage & -0.1097 & 0.0204 & -5.3775 & $<0.001$ & 0.8961 \\
Antenatal check-up & -0.3105 & 0.0493 & -6.2982 & $<0.001$ & 0.7331 \\
Place of work & 0.2732 & 0.0485 & 5.6330 & $<0.001$ & 1.3142 \\
Any miscarriage or abortion & 0.1906 & 0.0399 & 4.7769 & $<0.001$ & 1.2099 \\
\hline
\end{tabular}

Chi-Square Value $=678.2057(\mathrm{p}$-value $<0.001)$; Kappa-like statistic $=0.614$.

Table 5. SS-GEE estimates for pregnancy complications under the assumption of random effect structure within the responses.

\begin{tabular}{cccccc}
\hline Covariate & Coefficient & Standard error & Wald Statistic & p-value & Odds ratio \\
\hline Constant & -0.0452 & 0.0164 & -2.7561 & 0.006 & 0.9559 \\
Economic status & 0.5104 & 0.0572 & 8.9231 & $<0.001$ & 1.6659 \\
Age at marriage & -0.1197 & 0.0199 & -6.0151 & $<0.001$ & 0.8872 \\
Antenatal check-up & -0.3408 & 0.0472 & -7.2203 & $<0.001$ & 0.7112 \\
Place of work & 0.2537 & 0.0263 & 9.6464 & $<0.001$ & 1.2888 \\
Any miscarriage or abortion & 0.1893 & 0.0316 & 5.9905 & $<0.001$ & 1.2084 \\
\hline
\end{tabular}

Chi-Square Value $=928.2137(\mathrm{p}$-value $<0.001) ;$ Kappa-like statistic $=0.713$. 
From Table 5, we observe that economic status, age at marriage, antenatal check-up, place of work, and any miscarriage or abortion are significant at 0.01 level. The prevalence of the complications appears to be significantly higher among the respondents of better economic status. The odds ratio shows that there is about 67 percent increase in the risk of suffering from pregnancy complications among the women of higher economic status as compared to those of lower economic status. It is noteworthy that women with age at marriage more than 15 years are less likely (one-tenth) to experienced pregnancy complications as compared to their less than 16 years of age at marriage counterparts. It is startling that the prevalence of complications is reportedly higher during the initial stage of pregnancy and decreases significantly at the advanced stage. It is observed that there are 29 percent decrease in the risk of suffering from pregnancy complications among the reproductive women who have visited for antenatal check-up to that of women who are not taking antenatal check-ups.

Women who work outside of home are 1.29 times likely to encounter one of the above complications as compared to their homely counterparts. As compared the respondents with no miscarriage or abortion, pregnancy complications increase substantially (about 21 percent) for respondents having miscarriage(s) or abortion(s). Table 5 also represents that all the covariates used in the present study are highly significant under the random effect assumption. In addition to the positive association between economic status and prevalence of pregnancy complications, we find that place of work, and any miscarriage or abortion exert positive effect and age at marriage, antenatal check-up have negative effects as well. However, these risks of prevailing the pregnancy complication among mothers are higher when we have considered independence (Table 3 ), and exchangeable correlation (Table 4) within the responses as compared to the random effect structure (Table 5) for almost all covariates under consideration. More specifically, risk of suffering pregnancy complication is more in independence structure than exchangeable correlation and again the later consideration giving more risk than random effect structure. The kappa-like statistic is good (0.41 - 0.60) for GEE model under independence correlation, excellent (0.61 - 1.00) for exchangeable correlation, and also excellent for random effect structure within the responses. But, the highest kappa-like statistic is observed for the later GEE model.

Finally, it has been observed that the estimates obtained under the assumption of random effect structure within the responses come up with smaller value of standard errors than the other structures. Table 6 presents relative efficiency factors of the parameters obtained under different link structures with respect to those obtained under independence correlation assumption. Moreover, we observed that except for the intercept of the GEE model, the estimates of parameters of all the variables under random effect structure are significantly more efficient as compared to exchangeable correlation assumption. Furthermore, the highest relative efficiency factors of the estimate under random effect structure 
Table 6. Relative efficiencies of the estimates obtained by GEE assuming exchangeable correlation, and random effect.

\begin{tabular}{ccc}
\hline Covariate & Exchangeable correlation & Random effect \\
\hline Constant & 2.0939 & 1.9024 \\
Economic status & 2.7850 & 4.0314 \\
Age at marriage & 1.2255 & 1.2563 \\
Antenatal check-up & 1.1075 & 1.1568 \\
Place of work & 2.1773 & 4.0152 \\
Any miscarriage or abortion & 1.3333 & 1.6835 \\
\hline
\end{tabular}

versus independence correlation for economic status is 4.03 and lowest relative efficiency factor has been observed 1.16 for antenatal check-up.

\section{Conclusions}

Pregnancy related complications are very common in the rural areas of Bangladesh. In this study an endeavor has been made to identify the factors associated with some life-threatening complications commonly confronted by the rural reproductive women during childbearing tenure utilizing random effect SS-GEE model. All the covariates included in this study have been found to be highly significant (1 percent) in both bivariate and multivariate analysis. The generalized estimating equations approach is employed in order to analyze the repeated observations during pregnancy emerging from a follow-up study. Women from lower socio-economic status are exposed to more physical labor during pregnancy, which might help them to avoid some of the complications. It is demonstrated by the model that the risk is significantly higher among the respondents who reported their age at marriage lower than 16 years. In other words, lower age at marriage has an accumulated effect in the subsequent pregnancies as well. This shows the health problems associated with marriage at very young ages in the rural areas of Bangladesh. Those who reported their age at marriage more than fifteen years are less likely to suffer from pregnancy complications during index pregnancy. Similarly, the results confirmed the belief that the risk of suffering from complications decreases with an increase in the duration of successive pregnancy. This might be attributable to the psychological factors associated with every pregnancy. There are some researchers that have been found similar pattern for prevalence of complications during pregnancy with economic status and age at marriage [27] [29] [40].

However, there is a negative association between age at marriage, and antenatal check up and prevalence of complications. In addition, when GEE model moves from independence to exchangeable correlation, and random effect structure, we observed decreased risks of pregnancy complications along with the higher value of kappa-like statistic and higher values of relative efficiency factors for the estimates of the parameters of all the covariates. This study recommends that the SS-GEE model with random effect structure indicates better 
predictors than the GEE model with different correlation (independence, and exchangeable) structures [26] [27] [29] [40]. Thus, the GEE model with random effect structure within the response for repeated observations is fitted well.

\section{Acknowledgements}

The authors are indebted to the Ford Foundation for funding the data collection on maternal morbidity in Bangladesh and also grateful to BIRPERHT for providing data to analyze. The authors are also deeply thankful to the reviewers and editor for their valuable comments and professional services which greatly improve the quality of this manuscript.

\section{Conflicts of Interest}

The authors declare no conflicts of interest regarding the publication of this paper.

\section{References}

[1] United Nations (2006) The Millennium Development Goals Report 2006. United Nations, New York.

[2] Rajia, S., Sabiruzzaman, M., Islam, M.K., Hossain, M.G. and Lestrel, P.E. (2019) Trends and Future of Maternal and Child Health in Bangladesh. PLOS ONE, 14, e0211875. https://doi.org/10.1371/journal.pone.0211875

[3] World Health Organization (2016) World Health Statistics 2016: Monitoring Health for the SDGs Sustainable Development Goals. World Health Organization, Geneva.

[4] United Nations (2019) The Sustainable Development Goals Report 2019, Department of Economic and Social Affairs, United Nations, New York.

[5] Collin, S.M., Anwar, I. and Ronsmans, C. (2007) A Decade of Inequality in Maternity Care: Antenatal Care, Professional Attendance at Delivery, and Caesarean Section in Bangladesh (1991-2004). International Journal for Equity in Health, 6, Article No. 9. https://doi.org/10.1186/1475-9276-6-9

[6] Choolani, M. and Ratnam, S.S. (1995) Maternal Mortality. Journal of the Indian Medical Association, 93, 36-40.

[7] Bloom, S., Lippeveld, T. and Wypij, D. (1999) Does Antenatal Care Make a Difference to Safe Delivery? A Study in Urban Uttar Pradesh, India. Health Policy \& Planning, 14, 34-38. https://doi.org/10.1093/heapol/14.1.38

[8] WHO (World Health Organization) and UNICEF (United Nations Children's Fund) (2003) Antenatal Care in Developing Countries: Promises, Achievements and Missed Opportunities: An Analysis of Trends, Levels, and Differentials 1990-2001. World Health Organization and United Nations Children's Fund, Geneva.

[9] Afsana, K. (2004) The Tremendous Cost of Seeking Hospital Obstetric Care in Bangladesh. Reproductive Health Matters, 12, 171-180. https://doi.org/10.1016/S0968-8080(04)24142-8

[10] Chowdhury, M.E., Ronsmans, C., Anwar, I., Gausia, K., Das-Gupta, S., Blum, L.S., Dieltiens, G., Marshall, T., Saha, S. and Borghi, J (2006) Equality in Use of Home-Based or Facility-Based Skilled Obstetric Care in Rural Bangladesh: An Observational Study. The Lancet, 367, 327-332.

https://doi.org/10.1016/S0140-6736(06)68070-7 
[11] Pitchforth, E., Teijlingen, E.V., Graham, W.V., Dixon-Woods, M. and Chowdhury, M. (2006) Getting Women to Hospital in Not Enough: A Qualitative Study to Access to Emergency Obstetric Care in Bangladesh. BMJ Quality \& Safety, 15, 214-219. https://doi.org/10.1136/qshc.2005.017285

[12] Islam, M.R. and Odland, J.O. (2011) Determinants of Antenatal and Postnatal Care Visits among Indigenous People in Bangladesh: A Study of the Mru Community. Rural and Remote Health, 11, 1672-1680. https://doi.org/10.22605/RRH1672

[13] Goodburn, E.A., Gazi, R. and Chowdhury, M. (1995) Beliefs and Practices Regarding Delivery and Postpartum Maternal Morbidity in Rural Bangladesh. Studies in Family Planning, 26, 22-32. https://doi.org/10.2307/2138048

[14] National Institute of Population Research and Training (NIPORT), ORC Macro, Johns Hopkins University and International Centre for Diarrhoeal Disease Research, Bangladesh (ICDDR, B) (2003) Bangladesh Maternal Health Services and Maternal Mortality Survey 2001. National Institute of Population Research and Training, Dhaka; ORC Macro, Calverton; Johns Hopkins University, Baltimore; Centre for Health and Population Research, Dhaka.

[15] Gill, Z. and Ahmed, J.U. (2004) Experience from Bangladesh: Implementing Emergency Obstetric Care as Part of the Reproductive Health Agenda. International Journal of Gynecology and Obstetrics, 85, 213-220.

https://doi.org/10.1016/j.ijgo.2004.01.004

[16] Fauveau, V., Wojtyniak, B. and Chakraborty, J. (1989) Epidemiology and Cause of Deaths among Women in Rural Bangladesh. International Journal of Epidemiology, 18, 139-145. https://doi.org/10.1093/ije/18.1.139

[17] Koenig, M., Fauveau, V., Chowdhury, A.I., Chakraborty, J. and Khan, M.A. (1988) Maternal Mortality in Matlab, Bangladesh: 1976-85. Studies in Family Planning, 19, 69-80. https://doi.org/10.2307/1966492

[18] Rochat, R.W., Jabeen, S., Rosenberg, M.J., Measham, A.R., Khan, A.R., Obaidullah, M. and Gould, P. (1981) Maternal and Abortion Related Deaths in Bangladesh, 1978-1979. International Journal of Gynecology and Obstetrics, 19, 155-164. https://doi.org/10.1016/0020-7292(81)90056-4

[19] Rush, D. (2000) Nutrition and Maternal Mortality in the Developing World. American Journal of Clinical Nutrition, 72, 212S-240S. https://doi.org/10.1093/ajcn/72.1.212S

[20] Bhatia, J.C. (1995) Levels and Determinants of Maternal Morbidity: Results from a Community Based Study in India. International Journal of Gynecology \& Obstetric, 50, S153-S163. https://doi.org/10.1016/0020-7292(95)02504-6

[21] Joe, H. (1997) Multivariate Dependence Concept. Chapman \& Hall, London.

[22] Zeger, S.L. and Liang, K. (1986) Longitudinal Data Analysis for Discrete and Continuous Outcomes. Biometrics, 42, 121-130. https://doi.org/10.2307/2531248

[23] Liang, K. and Zeger, S. L. (1986) Longitudinal Data Analysis Using Generalized Linear Models. Biometrika, 73, 13-22. https://doi.org/10.1093/biomet/73.1.13

[24] Akanda, M.A.S., Jahan, K., Khanam, M. and Islam, M.A. (2005) Generalized Estimating Equations for Conditional and Unconditional Residuals in Diabetes and Mellitus Data. Journal of Applied Sciences, 5, 1228-1231. https://doi.org/10.3923/jas.2005.1228.1231

[25] Akanda, M.A.S., Khanam, M. and Islam, M.A. (2005) Comparison of Goodness-of-Fit Tests for GEE Modeling with Binary Responses to Diabetes and Mellitus. Journal of Applied Sciences, 5, 1214-1218.

https://doi.org/10.3923/jas.2005.1214.1218 
[26] Akanda, M.A.S. and Khanam, M. (2007) Fitting GEE Models and Goodness-of-Fit Tests for Delivery Complications in Rural Bangladesh. Australian Journal of Basic and Applied Sciences, 1, 431-440.

[27] Latif, A.H.M.M., Hossain, M.Z. and Islam, M.A. (2008) Model Selection Using Modified Akaike's Information Criterion: An Application to Maternal Morbidity. Austrian Journal of Statistics, 37, 175-184. https://doi.org/10.17713/ajs.v37i2.298

[28] Akhter, H.H., Chowdhury, M.E. and Sen, A. (1996) A Cross-Sectional Study on Maternal Morbidity in Bangladesh. Bangladesh Institute of Research for Promotion of Essential and Reproductive Health and Technologies (BIRPERHT), Dhaka.

[29] Gulshan, J., Chowdhury, R.I., Islam, M.A. and Akther, H.H. (2005) GEE Models for Maternal Morbidity in Rural Bangladesh. Austrian Journal of Statistics, 34, 295-304. https://doi.org/10.17713/ajs.v34i3.419

[30] Bates, D., Mächler, M., Bolker, B. and Walker, S. (2015) Fitting Linear Mixed-Effects Models Using lme4. Journal of Statistical Software, 67, 1-48. https://doi.org/10.18637/jss.v067.i01

[31] Pinheiro, J., Bates, D., DebRoy, S., Sarkar, D. and R Core Team (2018) nlme: Linear and Nonlinear Mixed Effects Models. R Package Version 3.1-137. http://CRAN.R-project.org/package=nlme

[32] Pinheiro, J.C. and Bates, D.M. (2000) Mixed-Effects Models in S and S-PLUS. Springer, New York.

[33] Diggle, P.J., Heagerty, P., Liang, K. and Zeger, S.L. (2002) Analysis of Longitudinal Data. 2nd Edition, Oxford University Press, Oxford.

[34] Qu, A., Lindsay, B. and Li, B. (2000) Improving Generalized Estimating Equations Using Quadratic Inference Function. Biometrika, 87, 823-836. https://doi.org/10.1093/biomet/87.4.823

[35] Gumedze, F.N. and Dunne, T.T. (2011) Parameter Estimation and Inference in the Linear Mixed Model. Linear Algebra and Its Applications, 435, 1920-1944. https://doi.org/10.1016/j.laa.2011.04.015

[36] Zeger, S.L., Liang, K. and Albert, P.S. (1988) Models for Longitudinal Data: A Generalized Estimating Equation Approach. Biometrics, 44, 1049-1060. https://doi.org/10.2307/2531734

[37] Fleiss, J.L. (1971) Measuring Nominal Scale Agreement among Many Raters. Psychological Bulletin, 76, 378-382. https://content.apa.org/doi/10.1037/h0031619

[38] Landis, R.J. and Koch, G.G. (1977) The Measurement of Observer Agreement for Categorical Data. Biometrics, 33, 159-174. https://doi.org/10.2307/2529310

[39] Williamson, J.N., Lin, H-M. and Barnhart, H.X. (2003) A Classification Statistic for GEE Categorical Response Models. Journal of Data Science, 1, 149-165.

[40] Salam, M.A. and Khanam, M. (2007) Fitting GEE Models and Goodness-of-Fit Tests for the Delivery Complications in Rural Bangladesh. Australian Journal of Basic and Applied Sciences, 1, 431-440. 